\title{
Continuous Probability Distributions over Second-Countable Spaces are Perfectly Supported
}

\author{
Yu-Lin Chou*
}

\begin{abstract}
We prove that every Borel probability measure over an arbitrary second-countable space vanishing at any singletons has support being a perfect set and being included in some co-countable perfect set. Thus the support of a continuous probability distribution over a second-countable space turns out to admit a richer structure.
\end{abstract}

MSC 2020: 60A10; 60E05; 03E15; 28A05

Keywords: continuous probability distributions; perfect sets; second-countable spaces; separable metric spaces; support of Borel probability measures

Throughout, a second-countable space is always Hausdorff. By slightly abusing language, a Borel probability measure (or simply a probability distribution) over a secondcountable space, in particular over a separable metric space, is called continuous if and only if its value is zero at every singleton. In a sense, working with second-countable spaces in the present context is the most general possible scenario. The theory of continuous probability distributions over Euclidean spaces $\mathbb{R}^{n}$ is well-developed and applied.

It is well-known (Theorem 2.1, Parthasarathy [2]) that every Borel probability measure $\mathbb{P}$ over a given separable metric space $\Omega$ may be uniquely assigned a "well-behaved" closed set, called the support $\operatorname{supp}(\mathbb{P})$ of $\mathbb{P}$, i.e. a unique closed subset $C$ of $\Omega$ such that i) $\mathbb{P}(C)=1$, ii) $C=\cap\{F \subset \Omega \mid F$ is closed, $\mathbb{P}(F)=1\}$, and iii) $C$ equals the set of all $x \in \Omega$ such that $G \in \mathscr{T}_{\Omega}(x)$ implies $\mathbb{P}(G)>0$; here $\mathscr{T}_{\Omega}(x)$ denotes the collection of all open subsets of $\Omega$ that contain $x$, that is, the collection of all neighborhoods of $x$. We extend the definition of support of Borel probability measures to second-countable spaces.

By a countable set we mean precisely a set that may be injected into $\mathbb{N} \equiv\{1,2, \ldots\}$. We show that the support of a continuous probability distribution over a given second-

*Yu-Lin Chou, Institute of Statistics, National Tsing Hua University, Hsinchu 30013, Taiwan, R.O.C.; Email: y.l.chou@gapp.nthu.edu.tw 
countable space is in fact also a perfect set, namely a closed set without isolated points, and is a subset of some co-countable perfect set:

Theorem. If $\Omega$ is second-countable, in particular if $\Omega$ is a separable metric space, and if $\mathbb{P}$ is a continuous probability distribution on the Borel sigma-algebra of $\Omega$, then $\operatorname{supp}(\mathbb{P})$ exists, is perfect, and is included in some co-countable perfect set.

Proof. We claim that $\Omega$ includes a co-countable perfect set. Indeed, the idea of CantorBendixson construction (e.g. Theorem 6.4, Kechris [1]) applies. Upon taking a countable basis $\mathscr{T}_{\Omega}^{0}$ for the given topology $\mathscr{T}_{\Omega}$ of $\Omega$, let

$$
\mathscr{V}:=\left\{V \in \mathscr{T}_{\Omega}^{0} \mid V \text { is countable }\right\} .
$$

Then $\cup \mathscr{V}$ is countable, and $F:=\Omega \backslash \cup \mathscr{V}$ is closed- $\mathscr{T}_{\Omega}$.

Since $\Omega$ is Hausdorff from assumption, every countable subset of $\Omega$ is a Borel subset of $\Omega$. On the other hand, the probability measure $\mathbb{P}$ is by assumption a continuous probability distribution, and hence $\mathbb{P}(A)=0$ for all countable $A \subset \Omega$. Since $\mathbb{P}(F)=1$, it follows that $F$ is an uncountable set. But then $F$ has no isolated points, i.e. $F$ is included in its derived set $\operatorname{cl}^{\prime}(F)$; for, if $x \in F \backslash \operatorname{cl}^{\prime}(F)$ then there is some uncountable $G \in \mathscr{T}_{\Omega}(x)$ such that $G \cap F=\{x\}$ and $G \cap(\Omega \backslash F) \subset \cup \mathscr{V} \backslash\{x\}$, which is impossible. Since $F$ is then perfect, the claim follows.

The proof of Theorem 2.1 in Parthasarathy [2] also serves second-countable spaces without changes, and so $\operatorname{supp}(\mathbb{P})$ exists (uniquely) and is such that

$$
\operatorname{supp}(\mathbb{P})=\Omega \backslash \cup\left\{G \in \mathscr{T}_{\Omega} \mid \mathbb{P}(G)=0\right\} .
$$

Since $\mathbb{P}(V)=0$ for all $V \in \mathscr{V}$, we have

$$
\operatorname{supp}(\mathbb{P}) \subset F .
$$

There remains to show that $\operatorname{supp}(\mathbb{P})$ is perfect. But if $x \in \operatorname{supp}(\mathbb{P})$ and $G \in \mathscr{T}_{\Omega}(x)$ with $G \cap \operatorname{supp}(\mathbb{P})=\{x\}$, then

$$
\mathbb{P}(G)=0+\mathbb{P}(G \cap(\Omega \backslash \operatorname{supp}(\mathbb{P})))=0
$$

by the assumed continuity of $\mathbb{P}$, which contradicts a defining property of $\operatorname{supp}(\mathbb{P})$. Now that $\operatorname{supp}(\mathbb{P})$ is perfect, the proof is complete.

\section{References}

[1] Kechris, A. S. (1995). Classical Descriptive Set Theory, Springer.

[2] Parthasarathy, K. R. (1967). Probability Measures on Metric Spaces, Academic Press. 